\title{
Peptostreptococcus stomatis sp. nov., isolated from the human oral cavity
}

\author{
Julia Downes and William G. Wade \\ King's College London Dental Institute, Infection Research Group, Floor 28, Guy's Tower, Guy's \\ Campus, London SE1 9RT, UK
}

Correspondence

William G. Wade

\begin{abstract}
Seven strains of anaerobic Gram-positive cocci isolated from human oral sites were subjected to a comprehensive range of phenotypic and genotypic tests. 16S rRNA gene sequence analysis revealed that the strains constituted a homogeneous group that was distinct from species with validly published names, but related to Peptostreptococcus anaerobius. All oral strains tested belonged to this group, whereas all non-oral strains studied were confirmed as $P$. anaerobius. A novel species, Peptostreptococcus stomatis sp. nov., is proposed to accommodate these oral strains. $P$. stomatis is weakly saccharolytic and produces acetic, butyric, isobutyric, isovaleric and isocaproic acids as end products of fermentation. The type strain of $P$. stomatis is W2278 $\left(=\mathrm{DSM} 17678^{\top}=\right.$ CCUG $51858^{\top}$ ); the $\mathrm{G}+\mathrm{C}$ content of the DNA of this strain is $36 \mathrm{~mol} \%$.
\end{abstract}

The anaerobic Gram-positive cocci comprise a diverse group of organisms. The majority of those associated with humans were formerly included within the genus Peptostreptococcus, but polyphasic taxonomic investigations have shown that they vary markedly in fundamental characteristics and number of new genera, including Anaerococcus, Finegoldia, Gallicola, 'Micromonas' and Peptoniphilus, have been proposed (Ezaki et al., 2001; Murdoch \& Shah, 1999).

Members of the type species of the genus Peptostreptococcus, Peptostreptococcus anaerobius, have been isolated from a wide range of human oral infections including periodontitis, dento-alveolar infections, pericoronitis, dentinal caries and endodontic infections (Moore et al., 1983; Sundqvist, 1992; Tanner et al., 1979). However, a specific PCR assay for the species (Riggio \& Lennon, 2002) failed to detect P. anaerobius in any of 60 subgingival plaque samples collected from subjects with periodontitis or 43 pus samples from dentoalveolar abscesses.

Molecular ecology studies characterizing the microflora associated with oral infections by $16 \mathrm{~S}$ rRNA gene sequence analysis have found a number of isolates and cloned $16 \mathrm{~S}$ rRNA genes with sequences identical to phylotype Peptostreptococcus CK035 (GenBank accession no. AF287763) (Munson et al., 2002; Paster et al., 2001), which is closely related to, but distinct from $P$. anaerobius. We hypothesize then, that $P$. anaerobius is not an organism found in the human mouth, but that those oral isolates previously

Published online ahead of print on 25 November 2005 as DOI 10.1099/ijs.0.64041-0.

Abbreviation: SPS, sodium polyanethol sulfonate.

The GenBank/EMBL/DDBJ accession number for the 16S rRNA gene sequence of Peptostreptococcus stomatis W2278 ${ }^{\top}$ is DQ160208. identified as $P$. anaerobius in fact belong to a closely related, as yet un-named, taxon, corresponding to phylotype Peptostreptococcus CK035.

The aim of this study was to characterize oral and non-oral isolates of $P$. anaerobius using a range of phenotypic and genotypic methods, to clarify the taxonomic position of this species and phylogenetically related taxa.

Strains W2175, W2205 and W2278 ${ }^{\mathrm{T}}$ were isolated from dento-alveolar abscesses, strains W3855 and W5396 were from endodontic infections, strain W5002 was from a periodontal pocket, strain W1948 was from a pericoronal infection and strain W3412 was from a leg ulcer. Strains AHC 14518 (isolated from a urinary tract infection), AHC 14540 (ankle wound), AHC 15114 (buttock abscess), AHC 50003 (vaginal infection) and AHC 50011 (leg ulcer) were the kind gift of E. Kononen, Anaerobe Reference Laboratory, Helsinki, Finland. The type strain of $P$. anaerobius (NCTC $11460^{\mathrm{T}}$ ) was obtained from the NCTC.

Strains were grown at $37^{\circ} \mathrm{C}$ on fastidious anaerobe agar (FAA; LabM) supplemented with $5 \%$ horse blood under anaerobic conditions $\left(80 \% \mathrm{~N}_{2}, 10 \% \mathrm{H}_{2}, 10 \% \mathrm{CO}_{2}\right)$ in an anaerobic workstation (Don Whitley Scientific). Colonial morphologies on this medium after 5 days incubation were viewed using a dissecting microscope. Cellular morphology was recorded after Gram-staining of smears prepared from 2-day-old FAA cultures.

Biochemical and physiological tests were performed as described previously (Downes et al., 2005). Enzyme profiles were generated with the Rapid ID 32A anaerobe identification kit (bioMérieux), according to the manufacturer's instructions, using bacteria harvested from blood agar plates (Columbia agar base; LabM) supplemented with $5 \%$ horse 


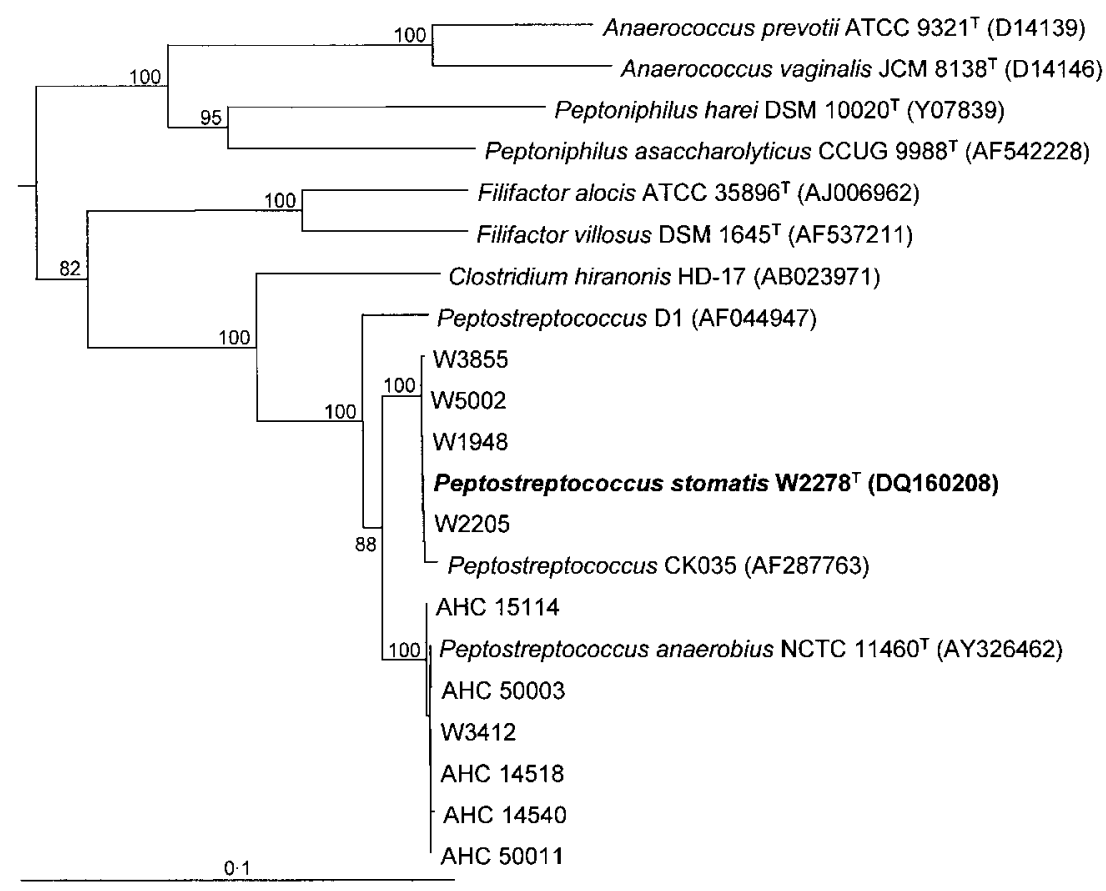

Fig. 1. Phylogenetic tree based on $16 \mathrm{~S}$ rRNA gene sequence comparisons over 1283 aligned bases showing relationships between strains of $P$. anaerobius and $P$. stomatis sp. nov., Peptostreptococcus phylotype CK035 and related species. The tree was constructed using the neighbour-joining method following distance analysis of aligned sequences. Numbers represent bootstrap values for each branch based on data for 100 trees. Accession numbers for $16 \mathrm{~S}$ rRNA gene sequences are given for each strain. Bar, $0 \cdot 1$ nucleotide substitutions per site. blood, and were performed in duplicate. Sensitivity to sodium polyanethol sulfonate (SPS) was determined by measuring the diameter of the zone of growth inhibition around an SPS disc (Oxoid) on a 3-day-old FAA plate culture.

The $\mathrm{G}+\mathrm{C}$ content of the DNA of strains $\mathrm{W} 2278^{\mathrm{T}}$ and W3855 was estimated by an HPLC method as described previously (Wade et al., 1999). A thermal denaturation method (Huß et al., 1983) was used to determine the extent of DNA-DNA hybridization between strains $\mathrm{W} 2278^{\mathrm{T}}$, W3855 and W3412 and the type strain of P. anaerobius, NCTC $11460^{\mathrm{T}}$. The 16S rRNA genes of the strains were sequenced as described previously (Downes et al., 2005). Sequences were assembled using the program BIOEDIT (Hall, 2004). Phylogenetic analysis was performed using the PHYLIP suite of programs (Felsenstein, 1993). Trees were constructed by three methods: a distance matrix was constructed using the Jukes-Cantor algorithm with DNADIST and NEIGHBOR was used to construct the tree by the neighbour-joining method; the maximum-likelihood method was used by means of DNAML and DNAPARS was used to construct a tree using a parsimony algorithm. Phylogenetic trees were viewed using TREEVIEW (Page, 1996).

Phylogenetic analysis of 16S rRNA gene sequences showed that the strains fell into two groups (Fig. 1). The three methods of tree construction produced trees with identical topology. The strains isolated from non-oral infections clustered with $P$. anaerobius NCTC $11460^{\mathrm{T}}$, whereas the oral strains formed a closely related but distinct cluster, which included oral phylotype Peptostreptococcus CK035. Members of the two groups each shared greater than $99.5 \%$ sequence identity with each other but strain W2278 ${ }^{\mathrm{T}}$, which was representative of the oral strains, shared only $97 \%$ sequence identity with strain NCTC $11460^{\mathrm{T}}$.

Variable region 1 of the $16 \mathrm{~S}$ rRNA gene was found to be entirely different in the two groups of strains, i.e. the loop in the secondary structure was 25 bases longer in the oral strains than the non-oral strains (Fig. 2). Interestingly, the specific forward primer described by Riggio \& Lennon (2002) was designed to anneal to this region, making it
Fig. 2. 16S rRNA gene sequence alignment of variable region 1 for strains of $P$. anaerobius and $P$. stomatis sp. nov., and the $P$. anaerobiusspecific PCR primer $(F)$ described by Riggio \& Lennon (2002). 
specific for $P$. anaerobius, but not Peptostreptococcus CK035. This further supports the hypothesis that $P$. anaerobius is not found in the human mouth.

The seven oral strains were obligately anaerobic, Grampositive cocci, $0.8 \times 0.8-0.9 \mu \mathrm{m}$, and arranged in pairs and short chains. After 5 days incubation on FAA plates, colonies were $0.8-1.8 \mathrm{~mm}$ in diameter, circular, entire, high convex to pyramidal, opaque, shiny and cream to off-white in colour with a narrow, grey, peripheral outer ring. In contrast, colonies of the seven non-oral $P$. anaerobius strains were $2 \cdot 2-4 \cdot 0 \mathrm{~mm}$ in diameter, circular, entire, convex, opaque, shiny and off-white in colour after 5 days incubation on FAA plates. Growth of all 14 oral and non-oral strains in peptone/yeast extract (PY) broth resulted in a clear broth with a sediment that produced a moderately turbid suspension on shaking ( 2 to $3+$, on a scale of 0 to $4+$ ). Growth was enhanced by the addition of $1 \%$ fermentable carbohydrates $(3$ to $4+)$. Fructose, glucose and maltose were weakly fermented by all strains, with terminal $\mathrm{pH}$ readings of $5 \cdot 3-5 \cdot 8$, whereas arabinose, cellobiose, lactose, mannitol, mannose, melezitose, melibiose, raffinose, rhamnose, ribose, salicin, sorbitol, sucrose and trehalose were not fermented. Major amounts of acetic and isocaproic acids, minor amounts of isobutyric and isovaleric acids and trace to minor amounts of butyric acid were produced as end products of metabolism in PY and peptone/yeast extract/ glucose (PYG) broth, with isobutyric and isovaleric acids more pronounced in PY than PYG broth. All strains produced a characteristic sweet pungent odour. None of the strains hydrolysed aesculin, arginine, gelatin or urea, nitrate was not reduced and catalase and indole were not produced.

The diameter of the zone of growth inhibition around the SPS discs was $15-17 \mathrm{~mm}$ for the seven non-oral P. anaerobius strains and $19-25 \mathrm{~mm}$ for the seven oral strains. The seven oral strains were positive for $\alpha$-glucosidase in the Rapid ID 32A identification panel, whereas all other reactions were negative, resulting in a profile of 0400000000 . The seven non-oral $P$. anaerobius strains, including the type strain P. anaerobius NCTC $11460^{\mathrm{T}}$, were positive for proline arylamidase in addition to $\alpha$-glucosidase resulting in a profile of 0400020000 . The $\mathrm{G}+\mathrm{C}$ content of the DNA of oral strains $\mathrm{W} 2278^{\mathrm{T}}$ and $\mathrm{W} 3385$ was estimated to be $36 \mathrm{~mol} \%$.

DNA-DNA hybridization between oral strains $\mathrm{W} 2278^{\mathrm{T}}$ and W3855 was determined to be $97 \%$. Hybridization between $P$. anaerobius NCTC $11460^{\mathrm{T}}$ and strains $\mathrm{W} 2278^{\mathrm{T}}$ and $\mathrm{W} 3855$ was 8 and $14 \%$, respectively. Hybridization between $P$. anaerobius NCTC $11460^{\mathrm{T}}$ and W3412 was $93 \%$.

The oral strains studied here constitute a homogeneous group and are clearly distinct from any species with validly published names. The name Peptostreptococcus stomatis sp. nov. is therefore proposed for these strains. Phenotypic characteristics that differentiate $P$. stomatis from $P$. anaerobius are shown in Table 1 and include colony morphology and the production of proline arylamidase by $P$. anaerobius, but not by $P$. stomatis. Sensitivity to SPS (diameter $\geqslant 12 \mathrm{~mm}$ ) has been used to differentiate $P$. anaerobius from other anaerobic Gram-positive cocci that are resistant to SPS (diameter $<12 \mathrm{~mm}$ ). P. stomatis strains are also sensitive to SPS and the seven strains in this study exhibited larger zones of growth inhibition than the $P$. anaerobius strains tested. Further strains of $P$. stomatis would need to be studied to establish whether the diameter of growth inhibition around an SPS disc is a reliable test to distinguish between $P$. stomatis and P. anaerobius.

\section{Description of Peptostreptococcus stomatis sp. nov.}

Peptostreptococcus stomatis (sto.ma'tis. N.L. gen. n. stomatis of the mouth from Gr. n. stoma mouth).

The description is based on seven strains isolated from the human oral cavity. Cells are obligately anaerobic, Grampositive cocci $(0.8 \times 0.8-0.9 \mu \mathrm{m})$ occurring in pairs and short chains. After 5 days incubation on FAA plates, colonies are $0.8-1.8 \mathrm{~mm}$ in diameter, circular, entire, high convex to pyramidal, opaque, shiny and cream to off-white in colour with a narrow, grey, peripheral outer ring. Moderate growth is obtained in broth media and growth is further enhanced by the addition of fermentable carbohydrates. Cells are weakly saccharolytic and ferment fructose, glucose and maltose weakly; arabinose, cellobiose, lactose, mannitol, mannose, melezitose, melibiose, raffinose, rhamnose, ribose, salicin, sorbitol, sucrose and trehalose are not fermented. Major amounts of acetic and isocaproic acids, minor amounts of isobutyric and isovaleric acids and trace to minor amounts of butyric acid are produced as end products of metabolism in PYG. Aesculin, arginine, gelatin and urea are not hydrolysed. Indole and catalase are not produced and nitrate is not reduced. The $\mathrm{G}+\mathrm{C}$ content of the DNA of the type strain is $36 \mathrm{~mol} \%$.

The type strain is $22278^{\mathrm{T}}\left(=\mathrm{DSM} \quad 17678^{\mathrm{T}}=\mathrm{CCUG}\right.$ $51858^{\mathrm{T}}$ ), isolated from infections of the human oral cavity.

Table 1. Differential characteristics of $P$. stomatis sp. nov. and $P$. anaerobius

SPS diameter is the diameter of growth inhibition around an SPS disc. Colony properties were determined following 5 days incubation on FAA plus $5 \%$ horse blood. Proline arylamidase activity was determined with the Rapid ID32A identification panel (bioMérieux).

\begin{tabular}{|lcc|}
\hline Characteristic & $\begin{array}{c}P \text {. stomatis } \\
(\boldsymbol{n}=7)\end{array}$ & $\begin{array}{c}\text { P. anaerobius } \\
(\boldsymbol{n}=7)\end{array}$ \\
\hline SPS diameter (mm) & $19-25$ & $15-17$ \\
Colony morphology & High convex/ & Convex \\
& pyramidal & \\
Colony diameter (mm) & $0 \cdot 8-1 \cdot 8$ & $2 \cdot 2-4 \cdot 0$ \\
Rapid ID32A profile & 0400000000 & 0400020000 \\
Proline arylamidase & - & + \\
& & \\
\hline
\end{tabular}




\section{Acknowledgements}

The assistance of Mrs Lynette Fernandes-Naglik in sequencing the $16 \mathrm{~S}$ rRNA genes of Peptostreptococcus anaerobius NCTC $11460^{\mathrm{T}}$ and Filifactor villosus DSM $1645^{\mathrm{T}}$ is gratefully acknowledged.

\section{References}

Downes, J., Sutcliffe, I., Tanner, A. C. R. \& Wade, W. G. (2005). Prevotella marshii sp. nov. and Prevotella baroniae sp. nov., isolated from the human oral cavity. Int J Syst Evol Microbiol 55, 1551-1555.

Ezaki, T., Kawamura, Y., Li, N., Li, Z. Y., Zhao, L. \& Shu, S. (2001). Proposal of the genera Anaerococcus gen. nov., Peptoniphilus gen. nov. and Gallicola gen. nov. for members of the genus Peptostreptococcus. Int J Syst Evol Microbiol 51, 1521-1528.

Felsenstein, J. (1993). PHYLIP (phylogeny inference package), version $3.5 \mathrm{c}$. Distributed by the author. Department of Genome Sciences, University of Washington, Seattle, USA.

Hall, T. (2004). BIOEDIT. Biological sequence alignment editor for Win95/98/NT/2K/XP. http://www.mbio.ncsu.edu/BioEdit/bioedit.html

Huß, V. A. R., Festl, H. \& Schleifer, K. H. (1983). Studies on the spectrophotometric determination of DNA hybridization from renaturation rates. Syst Appl Microbiol 4, 184-192.

Moore, W. E. C., Holdeman, L. V., Cato, E. P., Smibert, R. M., Burmeister, J. A. \& Ranney, R. R. (1983). Bacteriology of moderate (chronic) periodontitis in mature adult humans. Infect Immun 42, 510-515.
Munson, M. A., Pitt-Ford, T., Chong, B., Weightman, A. J. \& Wade, W. G. (2002). Molecular and cultural analysis of the microflora associated with endodontic infections. J Dent Res 81, 761-766.

Murdoch, D. A. \& Shah, H. N. (1999). Reclassification of Peptostreptococcus magnus (Prevot 1933) Holdeman and Moore 1972 as Finegoldia magna comb. nov. and Peptostreptococcus micros (Prevot 1933) Smith 1957 as Micromonas micros comb. nov. Anaerobe 5, 555-559.

Page, R. D. M. (1996). TREEVIEW: an application to display phylogenetic trees on personal computers. Comput Appl Biosci 12, 357-358.

Paster, B. J., Boches, S. K., Galvin, J. L., Ericson, R. E., Lau, C. N., Levanos, V. A., Sahasrabudhe, A. \& Dewhirst, F. E. (2001). Bacterial diversity in human subgingival plaque. J Bacteriol 183, 3770-3783.

Riggio, M. P. \& Lennon, A. (2002). Development of a PCR assay specific for Peptostreptococcus anaerobius. J Med Microbiol 51, 1097-1101.

Sundqvist, G. (1992). Associations between microbial species in dental root canal infections. Oral Microbiol Immunol 7, 257-262.

Tanner, A. C. R., Haffer, C., Bratthall, G. T., Visconti, R. A. \& Socransky, S. S. (1979). A study of the bacteria associated with advancing periodontitis in man. J Clin Periodontol 6, 278-307.

Wade, W. G., Downes, J., Dymock, D., Hiom, S. J., Weightman, A. J., Dewhirst, F. E., Paster, B. J., Tzellas, N. \& Coleman, B. (1999). The family Coriobacteriaceae: reclassification of Eubacterium exiguum (Poco et al. 1996) and Peptostreptococcus heliotrinreducens (Lanigan 1976) as Slackia exigua gen. nov., comb. nov. and Slackia heliotrinireducens gen. nov., comb. nov., and Eubacterium lentum (Prevot 1938) as Eggerthella lenta gen. nov., comb. nov. Int J Syst Bacteriol 49, 595-600. 